University of Nebraska - Lincoln

DigitalCommons@University of Nebraska - Lincoln

May 1996

\title{
Experimental and theoretical study of vibrations of a cantilevered beam using a $\mathrm{ZnO}$ piezoelectric sensor
}

B. R. Jooste

University of Nebraska - Lincoln

Hendrik J. Viljoen

University of Nebraska - Lincoln, hviljoen1@unl.edu

S. L. Rohde

University of Nebraska - Lincoln, srohde1@unl.edu

N. F. J. van Rensburg

University of Pretoria, South Africa

Follow this and additional works at: https://digitalcommons.unl.edu/cbmeviljoen

Part of the Chemical Engineering Commons

Jooste, B. R.; Viljoen, Hendrik J.; Rohde, S. L.; and van Rensburg, N. F. J., "Experimental and theoretical study of vibrations of a cantilevered beam using a ZnO piezoelectric sensor" (1996). Hendrik J. Viljoen Publications. 2.

https://digitalcommons.unl.edu/cbmeviljoen/2

This Article is brought to you for free and open access by the Chemical and Biomolecular Research Papers -- Faculty Authors Series at DigitalCommons@University of Nebraska - Lincoln. It has been accepted for inclusion in Hendrik J. Viljoen Publications by an authorized administrator of DigitalCommons@University of Nebraska - Lincoln. 


\title{
Experimental and theoretical study of vibrations of a cantilevered beam using a $\mathrm{ZnO}$ piezoelectric sensor
}

\author{
B. R. Jooste and H. J. Viljoen ${ }^{\text {a) }}$ \\ Department of Chemical Engineering, University of Nebraska-Lincoln, Lincoln, Nebraska 68588-0126 \\ S. L. Rohde ${ }^{\text {b) }}$ \\ Department of Mechanical Engineering and the Center for Materials Research and Analysis, \\ University of Nebraska-Lincoln, Lincoln, Nebraska 68588-0126 \\ N. F. J. van Rensburg \\ Department of Mathematics, University of Pretoria, Pretoria 0001, South Africa
}

(Received 16 October 1995; accepted 4 March 1996)

\begin{abstract}
Piezoelectric sensors can measure vibrations of solid structures very accurately. A model of a cantilevered beam, with a $\mathrm{ZnO}$ film on one side is presented. Both viscous and internal damping are considered. The output of the sensor is modeled and matched with experimental results by adjusting the damping parameters. A theoretical formulation for damage is introduced. Experimental results for a damaged beam confirm the shift in frequencies to lower values. The model is used to identify the extent of the damage. (C) 1996 American Vacuum Society.
\end{abstract}

\section{INTRODUCTION}

Transverse vibrations of cantilevered beams have been the subject of numerous studies. This system is more amenable to a mathematical analysis than more complex structures and has therefore served as basis for different studies in the fields of vibration and damage detection in structures. Banks and Inman ${ }^{1}$ used the cantilevered beam to study different damping mechanisms. They estimated the damping coefficients by using a least squares method on experimental time histories of beam vibrations. These damping coefficients were used in the analytical vibration model that was used to numerically simulate the dynamic response of the system and then compared to the experimental results. Rizos and Aspragathos ${ }^{2}$ used the cantilevered beam to study the inverse problem of crack location: determining the position and size of the crack from the sensor signal. This system also forms the basis for the development of materials with self-diagnostic capabilities (Viljoen and van Rensburg ${ }^{3}$ ). It is well known that local damage changes the natural vibration frequency of a cantilevered beam (cf. Adams et al. ${ }^{4}$ ). A very important aspect in the development of a self-diagnostic material is therefore accurate measurement of the natural frequencies.

Piezoelectric sensors in the form of a thin film could potentially be used as a sensing device by directly measuring the voltage output generated by the strained film. In studies on the use of natural frequencies to detect damage (Yuen, ${ }^{5}$ Adams et al., ${ }^{4}$ and Cawley and Adams ${ }^{6}$ ), the role of damping was not taken into account. Damping plays a very important role in natural vibrations since it has an influence on the natural frequencies, and even in the most simple form of damping (external viscous), the primary mode could become undetectable because it is overdamped. In contrast to viscous damping, strain rate damping (Kelvin-Voigt damping) at-

a) Author to whom correspondence should be addressed; electronic mail: chrdhjv@engvms.unl.edu

b)Electronic mail: srchde@unl.edu tenuates the different modes at different rates. Higher modes are damped out at a faster rate than lower modes. Banks and Inman ${ }^{1}$ also discussed two other forms of internal damping, namely, time hysteresis and spatial hysteresis. These two forms of damping do not permit analytical solutions, even for the cantilevered beam system, and one has to resort to numerical methods.

In this article we analyze the natural vibrations of a cantilevered beam with the consideration of both viscous and Kelvin-Voigt damping. Analytical solutions are obtained for the problem, which includes both damping mechanisms. Experimental measurements of a beam, coated with a thin $\mathrm{ZnO}$ layer that serves as the piezoelectric sensor, are compared with the theoretical results. A least squares method is used to identify the damping parameters and excellent agreement is found between theoretical and experimental values. A model for the description of damage is also presented and the theoretical model is used to quantify the extent of the damage.

\section{ZnO DEPOSITION}

A $\mathrm{ZnO}$ thin film was deposited onto a polished 304 stainless steel beam, measuring $145 \times 14.5 \times 0.89 \mathrm{~mm}$ by dc reactive magnetron sputtering for a period of $3 \mathrm{~h}$. See Table I for a summary of the deposition conditions. The sputtering was done in a mixed $\mathrm{Ar}-\mathrm{O}_{2}$ atmosphere using a bell-jar type dc magnetron sputtering system (Kurt J. Lesker Co.) with a zinc (99.9\% pure) target (disk) with a diameter of $50 \mathrm{~mm}$ and a thickness of $3.2 \mathrm{~mm}$. The substrate was positioned parallel to the target surface at a distance of about $88 \mathrm{~mm}$. No heating of the substrate, other than that caused by the sputtering process, was done during the $\mathrm{ZnO}$ film deposition, and no bias voltage was applied to the substrate. The sputtering system was evacuated to a base pressure of $2 \times 10^{-5}$ Torr by using a turbo pump backed by a roughing pump. No presputtering was done to remove the natural oxide layer on the target surface, since no shutter was available at the time. After the 
TABLE I. Deposition conditions.

\begin{tabular}{cc}
\hline \hline Item & Value \\
\hline Carrier gas & $\mathrm{Ar}$ \\
Substrate temperature & Ambient \\
Chamber pressure & $6-7 \mathrm{mTorr}$ \\
Substrate height & $85 \mathrm{~mm}$ \\
(above target) & \\
Plasma current & $40 \mathrm{~mA}$ \\
Deposition time & $3 \mathrm{~h}$ \\
$\mathrm{O}_{2}:$ Ar ratio & $1: 10$ \\
\hline
\end{tabular}

base pressure had been reached, argon (sputtering gas) and oxygen (reactive gas) were introduced to the vacuum chamber through two separate control valves. These valves were controlled by an MKS volumetric flowrate controller, which kept the flowrates at the predetermined optimum setpoints for the deposition of good quality $\mathrm{ZnO}$ films. After the pressure had stabilized at about $0.8 \mathrm{~Pa}$ (gauge pressure), the sputtering was started with the sputtering current controlled at 40 $\mathrm{mA}$. The film adherence was found to be very good and no problems with peeling were experienced.

\section{VIBRATION MEASUREMENTS}

The piezoelectric properties of $\mathrm{ZnO}$ film enable it to act as a sensor (when the film experiences strain, a voltage difference is created across the film) and also as an actuator (when an electric field is applied across the film, it is deformed). For the purpose of the vibration measurements, only the sensing ability of the film was used. One end of the stainless steel beam with the $\mathrm{ZnO}$ film sputtered onto one side was clamped, while the other end was left free to vibrate. A patch of carbon fibers was glued onto the $\mathrm{ZnO}$ film with an epoxy glue to serve as an electrode at the top of the $\mathrm{ZnO}$ film. The stainless steel beam itself served as the electrode at the bottom of the film (see Fig. 2 for the experimental setup). These two electrodes were connected to a digital oscilloscope to measure the difference in voltage across the film. The beam was given an initial displacement of about $75 \mathrm{~mm}$ at its free end and released to vibrate at its natural frequency. The voltage signal initiated by the vibration was captured on the oscilloscope and compared to the theoretical signal predicted by the model. The signal clearly shows the primary vibration mode of the beam with the secondary and tertiary vibration modes superimposed on it (see Fig. 3). It is also clear (see Fig. 3) that the tertiary mode is damped out after a few oscillations.

\section{FILM CHARACTERIZATION}

The $\mathrm{ZnO}$ film was characterized by analyzing its crystallinity and crystallographic orientation using $\mathrm{x}$-ray diffraction (XRD). It is known that crystalline $\mathrm{ZnO}$ with a hexagonal wurtzite crystal structure (and $6 \mathrm{~mm}$ symmetry) is an $n$-type semiconductor. It has a preferential crystallographic orientation of (0002) and is piezoelectric (Aeugle et al. ${ }^{7}$ ). $\mathrm{ZnO}$ exhibits strong self-texturing even when deposited onto amorphous substrates and at room temperature (Yamamoto

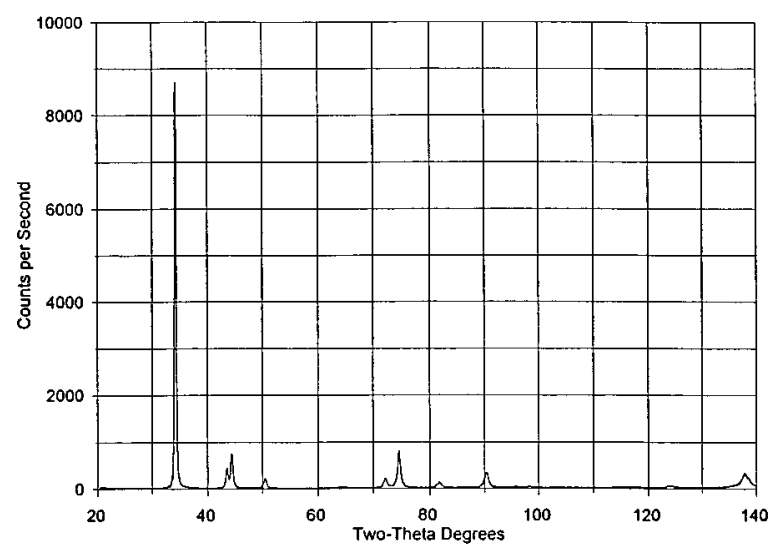

FIG. 1. XRD diagram of $\mathrm{ZnO}$ film sputtered on stainless steel substrate.

et al. ${ }^{8}$ ). The XRD analysis of the film showed a sharp (0002) diffraction peak $\left(2 \theta=34.42^{\circ}\right)$ for the $\mathrm{ZnO}$ film, which implies that the film is highly crystalline and that the crystals are oriented with their $c$ axes perpendicular to the stainless steel substrate. The film is therefore expected to be piezoelectric. An XRD pattern of the film for the conditions listed in Table I is shown in Fig. 1.

\section{MODEL FOR A CANTILEVERED PLATE}

We only consider one-dimensional transverse vibrations. It is assumed that the plate can be modeled as a onedimensional continuum. Reismann ${ }^{9}$ showed that in the case of pure bending, the solution for the plate problem approaches the solution for a beam as the width becomes smaller. In this study, we consider a stainless steel beam with dimensions $L: W: H \quad$ (length:width:thickness) $=(0.145: 0.0145: 0.00089)$. Let $\lambda_{m}$ denote the mass per unit length of the plate $\left(\lambda_{m}=\rho W H\right)$ and $D$ the flexural stiffness with $D=E_{Y} I$, where $I=W H^{3} / 12$ (moment of inertia) and $E_{Y}$ is the Young's modulus for stainless steel 304. Defining the dimensionless variables $x=X / L, u=U / L$, and $\tau=t \sqrt{D / L^{4} \lambda_{m}}$, the transverse displacement is given by

$$
\frac{\partial^{2} u}{\partial \tau^{2}}+\alpha_{v d} \frac{\partial u}{\partial \tau}+\alpha_{\mathrm{KV} d} \frac{\partial^{5} u}{\partial \tau \partial x^{4}}=-\frac{\partial^{4} u}{\partial x^{4}} .
$$

The viscous damping parameter $\alpha_{v d}$ is defined in terms of the viscous damping coefficient $C$ as $C L^{2} / \sqrt{\lambda_{m} D}$; likewise, we define the Kelvin-Voigt damping parameter $\alpha_{\mathrm{KV} d}$ in terms of the Kelvin-Voigt damping coefficient $C_{K}$ as $C_{K} I / L^{2} \sqrt{\lambda_{m} D}$. The boundary conditions at the fixed end are

$$
u=\frac{d u}{d x}=0, \quad x=0,
$$

and at the free end

$$
\begin{aligned}
& \frac{\partial^{2} u}{\partial x^{2}}+\alpha_{\mathrm{KV} d} \frac{\partial^{3} u}{\partial x^{2} \partial \tau}=0, \quad x=1, \\
& \frac{\partial^{3} u}{\partial x^{3}}+\alpha_{\mathrm{KV} d} \frac{\partial^{4} u}{\partial x^{3} \partial \tau}=0, \quad x=1 .
\end{aligned}
$$


The solution can be written in the form

$$
u(x, \tau)=V(x) S(\tau) .
$$

Since the problem is linear and homogeneous, $S(\tau)$ must have the form

$$
S(\tau)=e^{\omega \tau}
$$

where $\omega$ is a complex value that is still to be determined. Substitution of the above form for $u(x, \tau)$ reduces Eq. (1) to an ordinary differential equation:

$$
\frac{d^{4} V(x)}{d x^{4}}\left(1+\alpha_{\mathrm{KV} d} \omega\right)+\left(\omega^{2}+\alpha_{v d} \omega\right) V=0 .
$$

The boundary conditions are

$$
\begin{aligned}
& V=\frac{d V}{d x}=0, \quad x=0, \\
& \frac{d^{2} V}{d x^{2}}=\frac{d^{3} V}{d x^{3}}=0, \quad x=1 .
\end{aligned}
$$

Since $\omega=-1 / \alpha_{\mathrm{KV} d}$ does not permit nontrivial solutions of Eqs. (1)-(4), we can define the complex number $Z$ $\left(\equiv|Z| e^{i \theta}\right)$ as follows:

$$
Z=-\frac{\omega^{2}+\alpha_{v d} \omega}{1+\alpha_{\mathrm{KV} d} \omega} \text {. }
$$

The general solution to Eq. (6) is

$$
V=\sum_{j=1}^{4} A_{j} e^{\lambda_{j} x}
$$

where $\lambda_{j}$ is given by (de Moivre's theorem)

$$
\lambda_{j}=|Z|^{1 / 4} e^{i[\theta+2 \pi(j-1)] / 4}
$$

and

$$
\theta=\arg (Z) .
$$

Applying the boundary conditions (7) and (8) to the solution in Eq. (10) gives the homogeneous system of equations

$$
M \bar{a}=\overline{0},
$$

where

$$
\bar{a}=\left[\begin{array}{l}
A_{1} \\
A_{2} \\
A_{3} \\
A_{4}
\end{array}\right]
$$

and $M$ is defined as

$$
M=\left[\begin{array}{cccc}
1 & 1 & 1 & 1 \\
\lambda_{1} & \lambda_{2} & \lambda_{3} & \lambda_{4} \\
\lambda_{1}^{2} e^{\lambda_{1}} & \lambda_{2}^{2} e^{\lambda_{2}} & \lambda_{3}^{2} e^{\lambda_{3}} & \lambda_{4}^{2} e^{\lambda_{4}} \\
\lambda_{1}^{3} e^{\lambda_{1}} & \lambda_{2}^{3} e^{\lambda_{2}} & \lambda_{3}^{3} e^{\lambda_{3}} & \lambda_{4}^{3} e^{\lambda_{4}}
\end{array}\right]
$$

A nontrivial solution will exist if and only if

$$
\operatorname{det} M=0 \text {. }
$$

The boundary conditions (7) and (8) are the same as for the case of no damping. Therefore, Eq. (11) can also be stated as

$$
\cos (\lambda) \cosh (\lambda)=-1 \text {. }
$$

There exists an infinite number of solutions to Eq. (12) [or Eq. (11)]. Let $\lambda^{k}$ denote the $k^{\text {th }}$ solution; hence,

$$
\begin{aligned}
V_{k}= & A_{1} \cos \left(\lambda^{k} x\right)+A_{2} \cosh \left(\lambda^{k} x\right)+A_{3} \sin \left(\lambda^{k} x\right) \\
& +A_{4} \sinh \left(\lambda^{k} x\right) .
\end{aligned}
$$

Note that $e^{\lambda_{1}^{k} x} \equiv \cos \left(\lambda^{k} x\right), e^{\lambda_{2}^{k} x} \equiv \cosh \left(\lambda^{k} x\right), e^{\lambda_{3}^{k}}$ $\equiv \sin \left(\lambda^{k} x\right), e^{\lambda_{4}^{k}} \equiv \sinh \left(\lambda^{k} x\right)$.

It follows from Eq. (9) that two solutions $\omega_{k 1}$ and $\omega_{k 2}$ are associated with $\lambda^{k}$ :

$$
\omega^{2}+\left[\alpha_{v d}+\alpha_{\mathrm{KV} d}\left(\lambda^{k}\right)^{4}\right] \omega+\left(\lambda^{k}\right)^{4}=0 .
$$

In general, we can write the eigenfunction for the transverse beam vibrations as

$$
u=\sum_{k=1}^{4} u_{k}=\sum_{k=1}^{4} V_{k}\left(e^{\omega_{k 1} \tau}+B_{k} e^{\omega_{k 2} \tau}\right) .
$$

The orthogonality of the eigenfunctions $V_{k}$ is trivial to prove and they are complete; hence, they can be used as a basis to approximate $u(0, x)$.

We assume that the initial state is the equilibrium solution for a constant load at the free end:

$$
\begin{aligned}
& u(0, x)=h\left(\frac{3}{2} x^{2}-\frac{1}{2} x^{3}\right), \\
& \frac{\partial u(0, x)}{\partial \tau}=0,
\end{aligned}
$$

where $h$ is a dimensionless displacement at the free end. The initial velocity is zero [Eq. (17)] and

$$
B_{k}=-\omega_{k 1} / \omega_{k 2} .
$$

For this choice of initial condition, the Fourier coefficients converge rapidly and the first four eigenmodes $(k=1, \ldots, 4)$ give a good approximation of the initial state. This model is used to approximate the transverse displacement of the beam undergoing free vibration after an initial displacement $h$.

\section{COMPARISON BETWEEN EXPERIMENTAL AND THEORETICAL RESULTS}

The damping parameters are not known a priori and there do not exist theoretical or empirical relations to estimate them. The best option is to compare our experimental and theoretical sensors' output and reconcile the two data sets by fitting the two unknown damping parameters using a least squares method. A similar approach was followed by Inman and Banks ${ }^{1}$ to identify the parameters of various damping mechanisms in composite beams. In order to do the parameter identification, it is necessary to model the piezoelectric behavior of the thin film. In the following section we will give a brief description of the model for the piezoelectric sensor. 


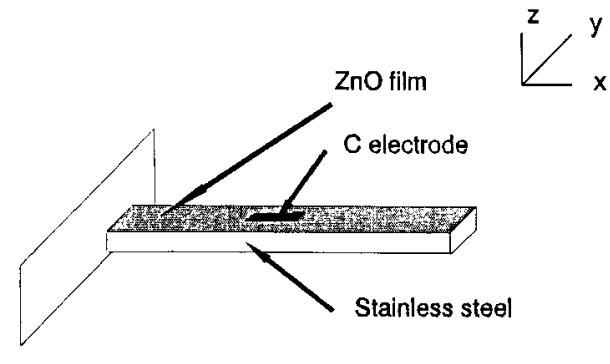

FIG. 2. Schematic of cantilevered beam with sensor.

\section{A. Model for sensor output}

When the plate is vibrating freely, the stress tensor is

$$
T=\left[\begin{array}{ccc}
T_{1} & 0 & T_{5} \\
0 & 0 & 0 \\
T_{5} & 0 & 0
\end{array}\right] .
$$

The strain tensor is given by $\left(\right.$ Auld $\left.^{10}\right)$

$$
S=\mathbf{s} T,
$$

where $\mathbf{s}$ is the compliance tensor for the beam. We assume that no slip occurs across the metal- $\mathrm{ZnO}$ interface. Therefore, strain is continuous.

In order to measure the electric field across the $\mathrm{ZnO}$ film, it is necessary to mount an electrode on top of it. A small rectangular section of the film is wetted with an epoxy resin and carbon fibers are placed on the resin, as shown in Fig. 2. The substrate (stainless steel 304) acts as the one electrode and the carbon fiber patch as the other electrode. Measuring from the fixed end, the electrode extends from $L_{1}$ to $L_{2}$. It is also assumed that no electric fields exist in the transverse directions of the $\mathrm{ZnO}$ film and the $c$ axis of the polycrystalline film is oriented in the direction normal to the beam. (This assumption is quite valid in light of the strong selftexturing of $\mathrm{ZnO}$ to grow with the $c$ axis normal on both amorphous and single crystal substrates.) Under open-circuit conditions, the following relationship holds for the $z$ component of the dielectric displacement vector $D$ (see Fig. 2 for axis orientation),

$$
\int D_{z} d A=0,
$$

where $\int d A$ is evaluated over the surface of the electrode. It follows from the relation between dielectric displacement, electric field, and strain,

$$
D_{z}=\epsilon_{z z}^{S} E_{z}+e_{z 1} S_{11}+e_{z 3} S_{33}+e_{z 1} S_{22}
$$

that

$$
\int E_{z} \epsilon_{z z}^{S} d A+\int\left(e_{z 1} S_{11}+e_{z 1} S_{22}+e_{z 3} S_{33}\right) d A=0 .
$$

Using an average electrical field strength $\bar{E}_{z}$, integration leads to the following equation:

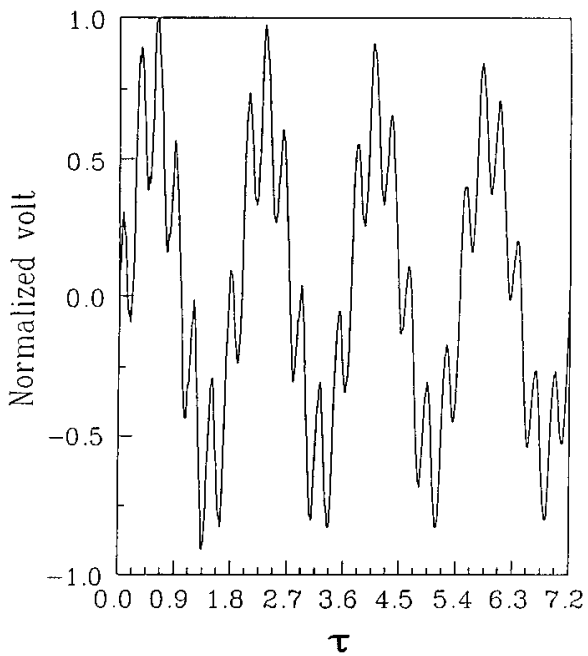

FIG. 3. Experimental output of sensor.

$$
\begin{aligned}
\bar{E}_{z} \epsilon_{z z}^{S} A= & -\int_{L_{1}}^{L_{2}}\left(e_{z 1} s_{11} \frac{d^{2} u}{d x^{2}} E_{Y} \frac{H}{2 L}+e_{z 1} s_{22} \frac{d^{2} u}{d x^{2}} E_{Y} \frac{H}{2 L}\right. \\
& \left.+e_{z 3} s_{33} \frac{d^{2} u}{d x^{2}} E_{Y} \frac{H}{2 L}\right) d x L_{3},
\end{aligned}
$$

where $L_{3}$ is the width of the electrode. This leads to an equation for the average electric field between the stainless steel beam and the carbon fiber electrode:

$$
\begin{aligned}
\bar{E}_{z}= & E_{Y} \frac{H}{2 L}\left[e_{z 1} s_{11}\left(\frac{d u}{d x}\right)_{L_{1}}^{L_{2}}+e_{z 1} s_{22}\left(\frac{d u}{d x}\right)_{L_{1}}^{L_{2}}\right. \\
& \left.+e_{23} s_{33}\left(\frac{d u}{d x}\right)\right] / \epsilon_{z z}\left(L_{2}-L_{1}\right) L_{3},
\end{aligned}
$$

and $d u / d x$ is obtained from the analytical solution (15). The permittivity and piezoelectric stress tensors are given by $\epsilon$ and $e$ respectively (see Auld ${ }^{10}$ ). If the film thickness is $h_{f}$, the signal output can be expressed in volts: $V=\bar{E}_{z} h_{f}$. This signal can then be compared with the experimental output.

\section{B. Evaluation of damping parameters}

It follows from Eq. (14) that the following relationship holds between $\alpha_{v d}$ and $\alpha_{\mathrm{KV} d}$ :

$$
\alpha_{v d}+\left(\lambda^{k}\right)^{4} \alpha_{\mathrm{KV} d}=-2 \omega_{R} .
$$

Note that $\omega=\omega_{R}+i \omega_{I}$ and $Z=\lambda^{4}$.

This linear relationship implies that we only have to fit one parameter. In Fig. 3 a normalized experimental sensor output is given for the beam used in this study. It was measured with an oscilloscope and the signal was transferred to a computer for further processing. A Fourier analysis was used to calculate the primary frequency $\omega_{I 1}=3.51595$. The first root of Eq. (12) is $\lambda_{1}=12.3624$, and using the relationship 


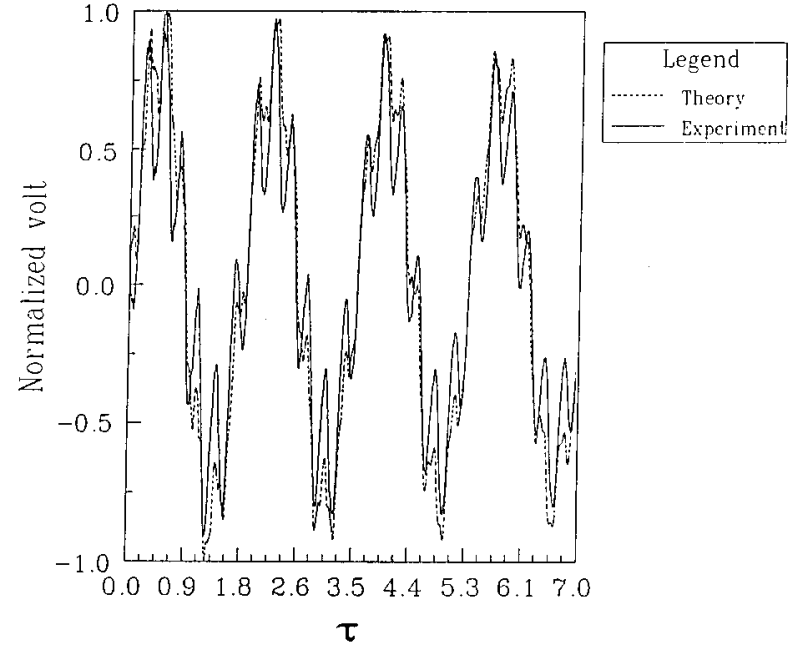

FIG. 4. Comparison between experimental and theoretical output.

$$
\lambda_{1}^{4}=\omega_{R}^{2}+\omega_{I}^{2}
$$

$\omega_{R}$ was used as -0.02144 . It is of paramount importance to evaluate $\omega_{I}$ accurately. The signal attenuation is determined by $\omega_{R}$, and it follows from Eq. (26) that inaccuracies in the evaluation of $\omega_{I}$ affect the value of $\omega_{R}$.

The linear relationship between $\alpha_{\mathrm{KV} d}$ and $\alpha_{v d}$ also provides upper bounds on the values of both damping parameters

$$
\begin{aligned}
& \alpha_{\mathrm{KV} d} \leqslant-2 \omega_{R} / \lambda_{1}^{4}, \\
& \alpha_{v d} \leqslant-2 \omega_{R} .
\end{aligned}
$$

In the absence of the internal damping mechanism, the wellknown result $\alpha_{v d}=-2 \omega_{R}$ is obtained. It can be concluded from Eq. (25) that an increase in the Kelvin-Voigt damping parameter leads to a decrease in the viscous damping parameter. It is known that higher frequencies are damped more strongly in the presence of Kelvin-Voigt damping, which is also quite apparent from Eq. (25).

The experimental and theoretical output are defined as $V_{E}(t)$ and $V_{T}(t)$, respectively. The function

$$
F\left(\alpha_{v d}\right)=\sqrt{\int_{t 1}^{t 2}\left[V_{E}(s)-V_{T}(s)\right]^{2} d s}
$$

is minimized when $\alpha_{v d}$ has the value $\alpha_{v d m}$. In Fig. 4 the experimental and theoretical curves are shown for $\alpha_{v d m}=0.04245$. The value for $\alpha_{\mathrm{KV} d}$ is $3.469 \times 10^{-5}$. The experimental output is simulated quite well by the model. Small discrepancies exist in the amplitude of the second eigenfunction. Excellent agreement is found between the experimental and model values of the higher frequencies. The system is now characterized.

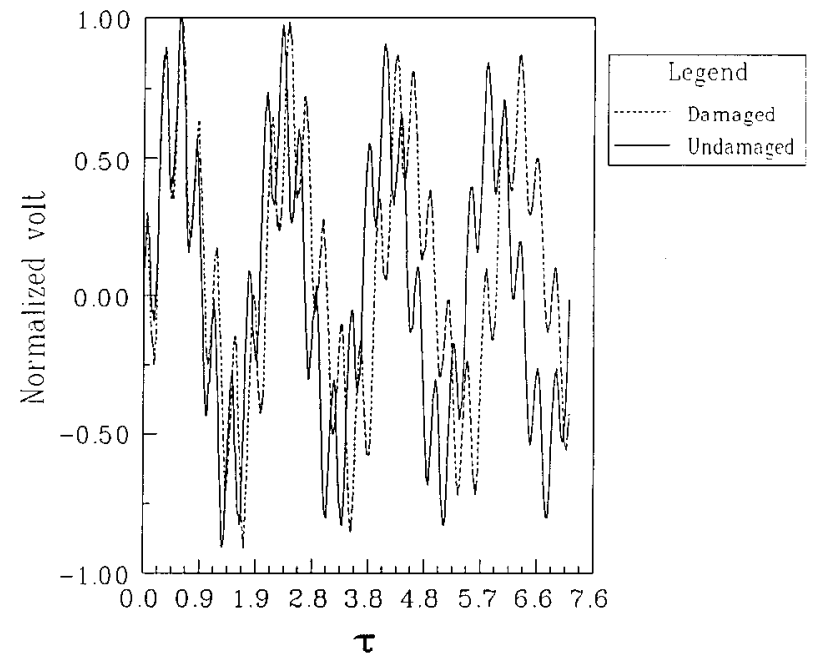

FIG. 5. Experimental output of a damaged and an undamaged beam.

\section{EXPERIMENTAL AND THEORETICAL ASSESSMENT OF DAMAGE}

Damage in the beam will be modeled as an elastic joint. Consider a local section of the plate, with length $l \ll L$. In this section, $D$ differs from the rest of the plate and is denoted as $D_{l}$. Define

$$
\delta=\lim _{l \rightarrow 0} \frac{l D}{D_{l} L}
$$

as a dimensionless measure of the magnitude of the damage. As a linear approximation, it can be shown that the following jump condition holds across $l$,

$$
\left[\frac{\partial U}{\partial x}\right]=\delta \frac{\partial^{2} U}{\partial x^{2}}
$$

$\left[\right.$ Note that $\left.[\cdot] \equiv(\cdot)^{+}-(\cdot)^{-} \cdot\right]$

In dimensionless form, the damage is located at $x=\gamma$. The conditions at the elastic joint are

$$
[V]=\left(\frac{d V}{d x}\right)-\delta \frac{d^{2} V}{d x^{2}}=\left(\frac{d^{2} V}{d x^{2}}\right)=\left(\frac{d^{3} V}{d x^{3}}\right)=0, \quad x=\gamma
$$

This definition of damage, which appears in the onedimensional model only pointwise, is not associated with a specific form of damage. Any damage such as delamination (for composite beams), cracks, or notches with a reduction in the moment of inertia or work-hardened area with different Young's modulus is included in this definition, provided that the domain of damage is small (i.e., $l \ll L$ ). Note that if this is not the case, then the beam can be modeled as three connected sections with different physical properties in the region associated with damage.

The problem can also be solved by separation of variables, with the additional complexity that different solutions exist on either side of $x=\gamma$. The existence condition [Eq. (12)] is augmented to include the continuity and jump conditions [Eq. (30)] at the elastic joint (see Viljoen and van Rensburg $^{3}$ for more details). However, we make the assump- 
tion that the damping parameters do not change in the presence of the elastic joint. When an elastic joint develops, it is associated with a decrease in the frequencies. This phenomenon is well known (cf. Adams et al. ${ }^{4}$ ). In Fig. 5 the experimental signal output of a damaged and undamaged beam is shown. In this particular case, the damage consisted of four holes $(1.5 \mathrm{~mm}$ diameter $)$ that were drilled in the beam at a distance $0.0109 \mathrm{~m}$ from the fixed end. The shift in frequencies is quite obvious.

In order to quantify the damage, we used the theoretical model with the damping parameters determined in the previous section. Using the parameter for damage to minimize the $L 2$ norm of the difference between the theoretical and experimental results, $\delta$ was found to be

$$
\delta=0.059
$$

\section{CONCLUSIONS}

The natural vibration of a stainless steel beam was studied by experimental and theoretical methods. A thin piezoelectric film of $\mathrm{ZnO}$ was sputtered on one side of the beam. The beam was clamped at one end and perturbed at the free end. A potential developed across the film during the vibration and it was measured. A mathematical model was also developed for this system and two forms of damping were considered, viscous damping and Kelvin-Voigt damping. The difference between the normalized theoretical and experimental output was minimized in the least square sense to find the viscous damping parameter. The damping parameters for this system are

$$
\begin{aligned}
& \alpha_{v d}=0.04245, \\
& \alpha_{\mathrm{KV} d}=3.469 \times 10^{-5} .
\end{aligned}
$$

Although the internal damping is small, it is an important mechanism in the damping of higher frequencies. The attenuation of the signal is determined by $\omega_{R}$, since

$$
\omega_{R}=\frac{-\left[0.04245+\left(\lambda^{k}\right)^{4} \times 3.469 \times 10^{-5}\right]}{2}
$$

scales with $\left(\lambda^{k}\right)^{4}$, higher frequencies are damped much faster.

The experimental output of a damaged beam was measured and compared with an undamaged beam and the sensor measured the shift in frequencies. A theoretical model of damage was also described and the signal output was calculated. The vibration of the beam was measured experimentally by the sensor and compared with the model sensor output. The parameter that quantifies the damage was calculated. Piezoelectric films of $\mathrm{ZnO}$ measure the frequencies of a vibrating beam very efficiently; it is sensitive towards structural changes in the beam and in combination with a theoretical model it can be used to determine and assess damage in structures.

\section{APPENDIX A: NOMENCLATURE}

$C$ : viscous damping coefficient $(\mathrm{kg} / \mathrm{ms})$.

$C_{K}: \quad$ Kelvin-Voigt damping coefficient $(\mathrm{kg} / \mathrm{ms})$.

s: $\quad$ compliance tensor $(1 / \mathrm{Pa})$.

$D: \quad$ plate flexural rigidity $E_{Y} I\left(\mathrm{~Pa} \mathrm{~m}^{4}\right)$.

$D_{z}: \quad z$ component of dielectric displacement vector $\left(\right.$ coulomb $\left./ \mathrm{m}^{2}\right)$.

$E_{z}: \quad z$ component of electric field $(\mathrm{V} / \mathrm{m})$.

$E_{Y}: \quad$ longitudinal Young's modulus $(\mathrm{Pa})$.

$e_{z i}$ : elements of $z$ component of piezoelectric stress tensor $\left(\right.$ coulomb $\left./ \mathrm{m}^{2}\right)$.

$H$ : $\quad$ thickness of plate (m).

$h$ : dimensionless initial displacement of free end.

$h_{f}$ : thickness of $\mathrm{ZnO}$ film (m).

I: $\quad$ moment of inertia $W H^{3} / 12\left(\mathrm{~m}^{4}\right)$.

$L: \quad$ length of plate $(\mathrm{m})$.

$L_{1,2}$ : position of electrode in the $x$ axis $(\mathrm{m})$.

$L_{3}: \quad$ width of electrode (m).

$S: \quad$ strain tensor.

$T: \quad$ stress tensor $(\mathrm{Pa})$.

$U: \quad$ transversal displacement (m).

$u: \quad$ dimensionless transverse displacement $(U / L)$.

$X$ : dimensional distance along the $x$ axis (m).

$x: \quad$ dimensionless distance along the $x$ axis.

$t: \quad$ time (s).

$V: \quad$ output of signal (V).

$V(x): \quad$ solution of Eq. (6).

$W: \quad$ width of plate $(\mathrm{m})$.

\section{APPENDIX B: GREEK SYMBOLS}

$\alpha_{v d}$ : dimensionless viscous damping coefficient, $C L^{2} / \sqrt{\lambda_{m} D}$.

$\alpha_{\mathrm{KV} d}$ : dimensionless Kelvin-Voigt damping coefficient, $C_{K} / L^{2} \sqrt{\lambda_{m} D}$.

$\epsilon_{z z}$ : normal $z$ component of permittivity tensor (farad/ $\mathrm{m})$.

$\lambda_{m}: \quad$ mass per unit length of plate $(\mathrm{kg} / \mathrm{m})$.

$\omega: \quad$ dimensionless frequency.

$\rho: \quad$ density of beam $\left(\mathrm{kg} / \mathrm{m}^{3}\right)$.

$\tau$. dimensionless time $t\left(\sqrt{D / L^{4} \lambda_{m}}\right)$.

${ }^{1}$ H. T. Banks and D. J. Inman, J. Appl. Mech. 58, 716 (1991).

${ }^{2}$ P. F. Rizos and N. Aspragathos, J. Sound Vibration 138, 381 (1990).

${ }^{3}$ H. J. Viljoen and N. F. J. van Rensburg, AIChE J. 42, 1101 (1996).

${ }^{4}$ R. D. Adams, P. Cawley, C. J. Pye, and B. J. Stone, J. Mech. Eng. Sci. 20, 93 (1978)

${ }^{5}$ M. M. F. Yuen, J. Sound Vibration 103, 301 (1985).

${ }^{6}$ P. Cawley and R. D. Adams, J. Strain Anal. 14, 49 (1979).

${ }^{7}$ T. H. Aeugle, H. Bialas, K. Heneka, and W. Pleyer, Thin Solid Films 201, 293 (1991)

${ }^{8}$ T. Yamamoto, T. Shiosaki, and A. Kawabata, J. Appl. Phys. 51, 3113 (1980).

${ }^{9}$ H. Reismann, Elastic Plates: Theory and Application (Wiley, New York, 1988).

${ }^{10}$ B. A. Auld, Acoustic Fields and Waves in Solids (Wiley, New York, 1982). 